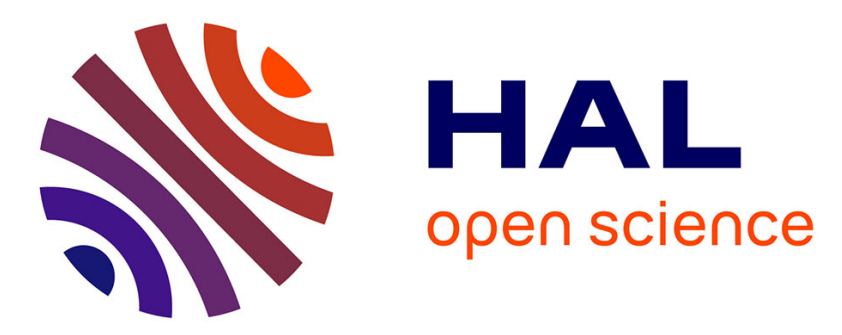

\title{
A survey on mobility management protocols in Wireless Sensor Networks based on 6LoWPAN technology
}

Maha Bouaziz, Abderrezak Rachedi

\section{To cite this version:}

Maha Bouaziz, Abderrezak Rachedi. A survey on mobility management protocols in Wireless Sensor Networks based on 6LoWPAN technology. Computer Communications, 2016, 74, pp.3-15. 10.1016/j.comcom.2014.10.004 . hal-01078082

\section{HAL Id: hal-01078082 \\ https://hal.science/hal-01078082}

Submitted on 27 Oct 2014

HAL is a multi-disciplinary open access archive for the deposit and dissemination of scientific research documents, whether they are published or not. The documents may come from teaching and research institutions in France or abroad, or from public or private research centers.
L'archive ouverte pluridisciplinaire HAL, est destinée au dépôt et à la diffusion de documents scientifiques de niveau recherche, publiés ou non, émanant des établissements d'enseignement et de recherche français ou étrangers, des laboratoires publics ou privés. 


\title{
A Survey on Mobility Management Protocols in Wireless Sensor Networks based on 6LoWPAN Technology
}

\author{
Maha Bouaziz ${ }^{\mathrm{a}}$, Abderrezak Rachedi ${ }^{\mathrm{b}}$ \\ ${ }^{a}$ University of Manouba \\ Hana Research Laboratory \\ Manouba, Tunisie \\ Email: maha_bouaziz@yahoo.fr \\ ${ }^{b}$ Université Paris-Est \\ LIGM (UMR8049), UPEM \\ F-77454, Marne-la-Vallée France \\ Email: rachedi@univ-mlv.fr
}

\begin{abstract}
Mobility has the advantage of enlarging WSN applications. However, proposing a mobility support protocol in Wireless Sensor Networks (WSNs) represents a significant challenge. In this paper, we propose a survey on the mobility management protocols in Wireless Sensor Networks based on 6LoWPAN technology. This technology enables to connect IP sensor devices to other IP networks without any need for gateways. We highlight the advantages and drawbacks with performances issues of each studied solution. Then, in order to select a typical classification of mobility management protocols in WSNs, we provide some classification criteria and approaches on which these protocols are based. Finally, we present a comparative study of the existing protocols in terms of the required performances for this network type.
\end{abstract}

Keywords: Wireless Sensor Network, 6LoWPAN, Mobility support protocols, Classification criteria, Comparative study.

\section{1. introduction}

Traditional Wireless Sensor Networks (WSNs) are developed using static nodes (SNs) [1][2][3][4]. These networks can be applied in numerous applications such as healthcare [5][6], military, industrial, monitoring, tracking based on multimedia sensor [7] and many other fields [8][9][10]. Hence, a lot of research and propositions are made for static scenarios. Nevertheless, the advanced technology involves applying more complex applications, which require mobility of its nodes [11]. Mobility of nodes can enlarge WSN applications [12]. Moreover, mobility can prolong the nodes lifetime, since data transfer between two nodes does not usually use the same relayed nodes in the path route. In addition, it serves to increase connectivity between nodes, since mobile nodes can help the communication between two isolated nodes [13]. It also helps to extend the coverage area of interest [14][15]. However, mobility can cause some challenging problems, like disconnection of nodes during the handover process, which causes data loss and negatively impact the performances of applications. Other issues related to the nodes mobility are resource management, topology control, routing protocol, quality of services and security.

In this paper, we focus on mobility management protocols in WSNs based on 6LowPAN technology [11][16][17]. This technology was proposed by IETF Working Group in order to introduce IPv6 over IEEE 802.15.4 [18][19][20][21]. The main aim of this technology was to propose an adaptation layer between the link layer and the network layer in order to face the problem related to disproportion of IPv6 packet size compared to IEEE 802.15.4 frame size [22][23]. Moreover, many header compression techniques have been proposed taking into account WSN characteristics like limited resources in terms of power, data rate, bandwidth, processing and storage capacities. The IEEE 802.15.4 standard has enabled to reduce power consumption in WSNs using a periodic sleep/wake-up process [24]. In addition, using IPv6 packets instead of IPv4 packets offered a more important address space, that helps to deploy an important number of nodes and satisfy scalability performance. Hence, introducing IPv6 over IEEE 802.15.4 made data accessible at any-time and from anywhere through the Internet. Therefore, 6LoWPAN offers the possibility to establish a direct connectivity between devices based on the IP address. Unlike ZigBee technology [25], each external communication from a WSN requires a Zigbee coordinator (ZC) or a gateway as an intermediate node which centralizes this kind of communication [26].

The aim of mobility support protocols is to keep nodes reachable and connected during the handover process, without interruption of connectivity [11]. Thus, when a node moves away from its neighbor's coverage, the protocol must rapidly provide an alternative router and ensure the configuration of a new interface for the mobile node.

The contribution of this paper is summarized as follows:

- Review of the state-of-the-art of mobility management protocols in Wireless Sensor Networks (WSNs) based on 6LoWPAN technology. The advantages and drawbacks 


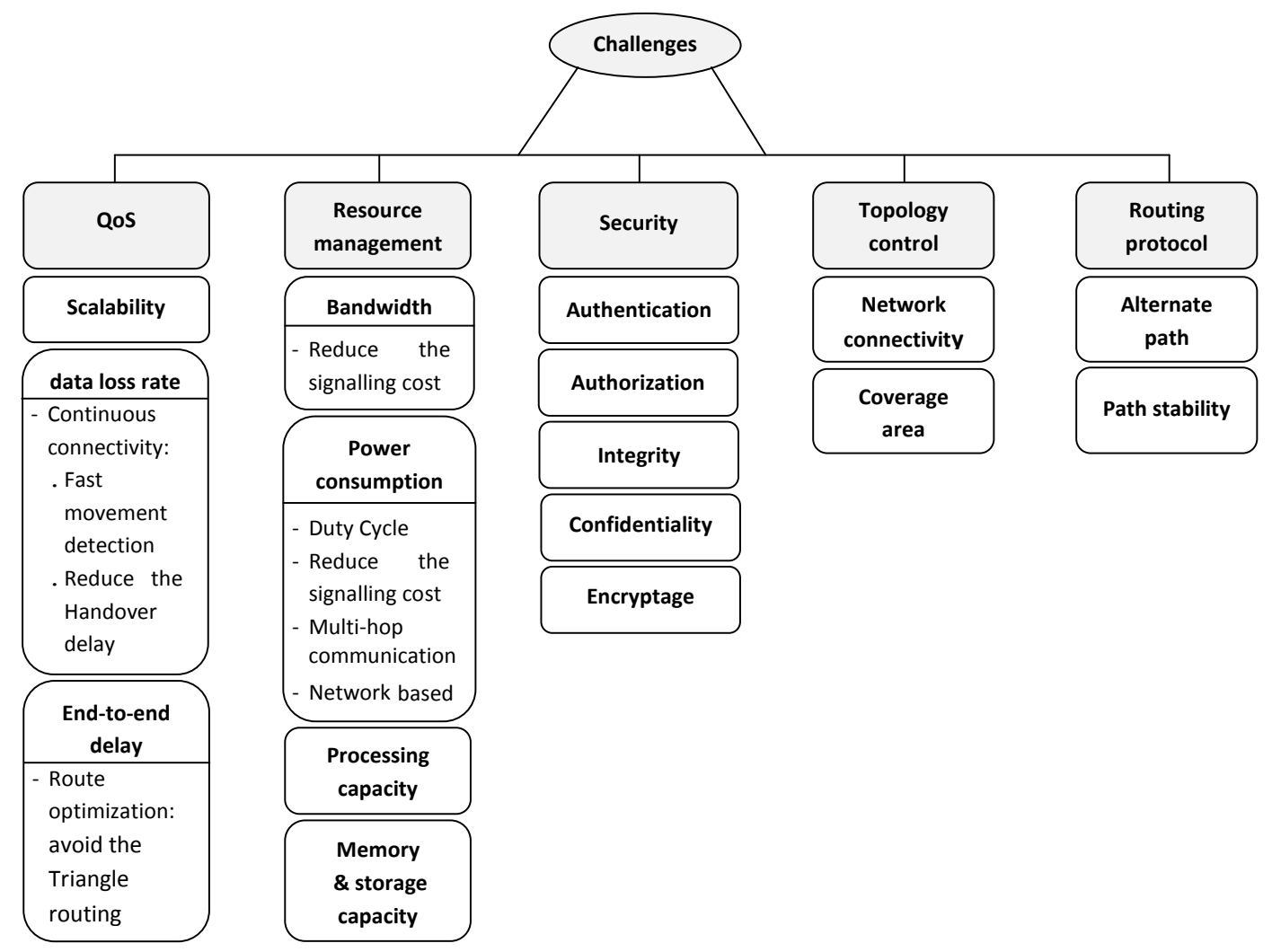

Figure 1: Challenge of mobility management for sensor Networks based on 6LoWPAN

with performance issues of each studied solution are highlighted.

- An attempt of mobility management protocols classification in WSN is proposed, after studying different criteria and approaches.

- A comparative study of existing mobility support protocols in WSN is proposed and analyzed.

The remainder of this paper is organized as follows: section 2 discussed the challenges to provide and design a protocol of mobility management. Section 3 focused on the classification criteria of existing mobility support protocols proposed for wireless networks, to select the best criteria which might be applied in 6LoWPAN Networks. Then, in section 4, we presented our comparative study considering the limited constraints of 6LoWPAN Networks. Section 5 discussed the future directions to be considered for the design of a mobility support protocol in the 6LoWPAN Networks. Finally, in section 6 concludes this paper by conclusion and perspectives.

\section{Mobility management, Challenges and Design Issues}

Mobility is the act of a node changing its attachment point due to the topology change. Before studying solutions dealing with mobility, we should understand its causes to be able to point out the appropriate challenge. In WSN based on 6LoWPAN technology, topology change is caused by some reasons such as physical movement, failure of some routers, using aggressive sleep, radio channel conditions since the radio propagation is affected by the environment change. Other possible reasons can be the network performances like the delay, the packet loss and the low signal that may cause a change in the topology [11].

The change of the attachment point requires disconnection of the mobile nodes. This disconnection causes significant problems of data loss and affects the proper functioning of applications. Besides, considering that WSN with 6LoWPAN technology imposes some delicate constraints and requirements [15], it has become urgent to discuss needed challenges to deal with these encountered problems as illustrated in Fig. 1.

In WSN with 6LoWPAN technology, the greatest challenge consists of providing a suitable "Quality-of-Service" (QoS) with different constraints consideration. For instance, mobility management must be efficient with an important density of nodes (i.e. ensure "scalability"). Moreover, mobility support protocol must mitigate the data loss rate. This problem occurs when the mobile node is disconnected during the handover process. Thus, it is important to reduce the handover delay in order to limit the disconnection time and a continuous connectivity. Furthermore, after the handover process, mobility management must keep the same end-to-end delay as used before this process. Hence, in 6LoWPAN technology, protocol must avoid 
the triangle routing ${ }^{1}$ (as illustrate in Fig. 2) which enlarge the needed delay to communicate between the mobile node and its correspondent, as it is used by some solutions such as "Hospital Wireless Sensor Networks" (HWSN6) [27][28][29], Inter-PAN [30][31] and "Low Mobility" (LoWMob)[32].

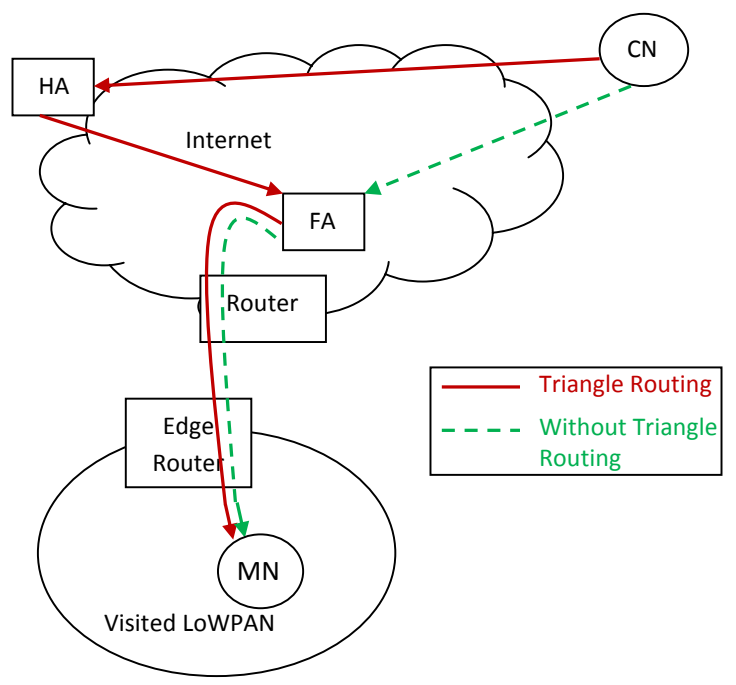

Figure 2: Communication between the mobile node and its correspondent through triangle routing process

On the other hand, the resources management is regarded as a significant challenge to design a protocol dealing with mobility [33]. This challenge arises because of the constraints and the limited resources of WSN with 6LoWPAN mainly in terms of power, bandwidth, memory and processing capacity. That's why, it is important to significantly reduce the cost of signaling messages, overhead communications and processing. Besides, the existing duty cycle used to reduce the energy consumption is not designed to support mobility constraints, thus, it must be adapted to this context.

The security issue is another challenge which must be taken into account in the mobility solution. In fact, the WSN nodes are exposed to attacks which disturb the mobility process by introducing false information. Thus, a trust model for a mobility scenario in WSN must be designed, and considered by the mobility management protocol to provide a secure network. The security services like authentication, authorization, integrity and confidentiality of data must be smartly introduced in mobile WSN [34]. The existing security mechanisms like intrusion detection systems must be adapted to support mobility in WSN [14].

The other challenges that must be considered are the topology control and the routing protocol. The topology control is conceived to improve the network connectivity, to increase the coverage of deployment area [35], and also to reduce the energy consumption and increase the networks lifetime [36][37].

\footnotetext{
${ }^{1}$ Communication between a mobile node and its correspondent: Packet from a correspondent node is forwarded to the Home Agent, then, to the Foreign Agent and finishes at the mobile node.
}

The performance of the routing protocol can directly impact the mobility management like re-activity to restore the link between two communicating nodes (time to find an alternative path when the intermediate nodes are not available), and the path stability [38].

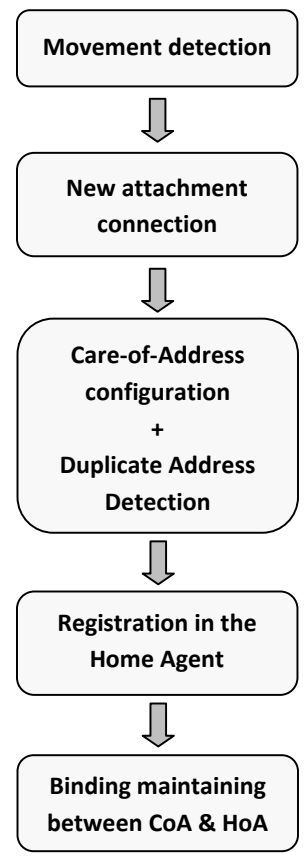

Figure 3: Operations of mobility support protocol for mobile Networks

Considering the cited challenges, operations of the mobility support protocol should take into account constraints and requirements of the 6LoWPAN technology. These operations follow some steps as shown in Fig. 3. The first step begins by detecting the movement of the node (or network). In 6LoWPAN network, it is necessary to achieve a good choice of the entity which performs the detection step, because of the limited resources capacity of the mobile nodes. In addition, it is interesting to perform movement detection with low resources consumption in order to satisfy the requirements and constraints of this network type. The second step, the Mobile Node (MN) performs a new address configuration called Care of Address (CoA), and then performs the Duplicate Address Detection (DAD). The third step is the registration in the Home Agent, which is carried out by sending a Binding Update (BU) having the new address to the Home Agent. According to the restriction resources of the mobile node in the 6LoWPAN technology, the protocol should consider the best choice of the entity which performs this task. The final step is performed by the Home Agent (HA), which maintains Binding between the two addresses (HoA and CoA) after receiving the binding update. Then, it buffers and forwards traffic between the mobile node and its correspondent. The proposed protocols to deal with mobility perform the cited operations with different ways, which generates diverse classifications presented in the next section. 


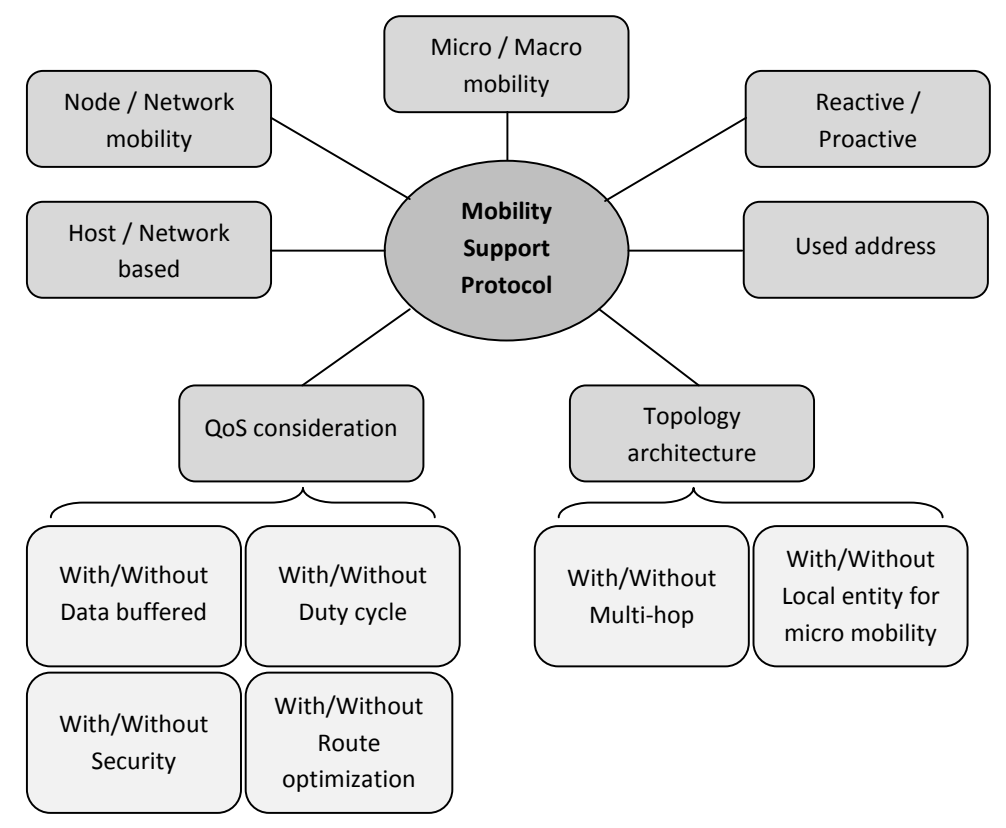

Figure 4: Classification criteria of mobility support protocols for 6LoWPAN Networks

\section{Classification criteria of mobility support protocols}

Protocols dealing with mobility in Wireless Sensor Network (WSN) based on 6LoWPAN technology should take into account not only the requirements of the application, but also WSN' characteristics. WSN has limited resources namely in terms of power, memory, processing capacity, bandwidth, short range, low data rate and small packet size.

In the following parts of this section, we define and discuss the potential classification criteria of mobility support protocols illustrated in Fig. 4.

\subsection{Node and Network mobility}

According to the application requirements, two classes of mobility can be considered: Node and network mobility. The "node mobility" refers to the mobility of only one node either in the same PAN or between different PANs, regardless to other nodes. It occurs as a result of an attachment change of the node in an independent way. In contrast, the "network mobility" refers to the mobility of the entire LoWPAN. In 6LoWPAN, such a network includes an edge router and member nodes, while only the edge router changes its attachment point on the Internet and the nodes remain attached to it [11]. This second class is a kind of the macro mobility type (explained in the next subsection).

\subsection{Macro and micro mobility}

According to the topology and application needs, two types of node mobility need to be taken into account. On the one hand, the "micro mobility", which refers to the node mobility within the same sensor network domain. In 6LoWPAN Networks, micro mobility is identified by the mobility of a node into the same LoWPAN domain, where the prefix remains unchanged. Thus, the mobility of such a node, changing its attachment point from an edge router to another within the same extended 6LoWPAN, is considered as a micro mobility. On the other hand, the "macro mobility" refers to the node mobility between different sensor Networks. In 6LoWPAN Networks, macro mobility is identified by the mobility of a node between different LoWPANs, where the prefix is changed.

Hence, protocol dealing with mobility for 6LoWPAN Networks must take into account these different mobility types, because of its impact on the prefix and then on the IPv6 address of the mobile nodes.

\subsection{Network and host based protocol}

Two kinds of protocols are distinguished: the first is called the "network based protocol", and the second is the "host based protocol" [34]. In the "network based protocol", the signaling messages, related to the movement detection and Binding Update $^{2}$, are sent by a static device in the network and not by the mobile sensor node. In literature, some existing solutions are based on this approach like Inter-PAN [30][31], Inter-Mario [39] and Cluster-Based Scheme [40]. In the "host based protocol", the mobile sensor node is involved in the signaling messages process. Some existing solutions based on this approach are proposed in "Mobile IPv6" (MIPv6)[41] and "Fast Handover for Mobile IPv6" (FMIPv6)[42].

According to WSN constraints (limited resources: power, processing, memory, and throughput), it is recommended to

\footnotetext{
${ }^{2}$ Message transmitted to the Home Agent to inform about the movement and the taken change
} 
perform the first kind (the network based protocol) for the reason to reduce the signaling cost and conserve power of mobile nodes [33][43].

\subsection{Reactive and proactive detection protocol}

Mobility support protocol can be classified into two categories:

- Reactive protocol: In this kind of protocol, dealing with mobility (such as movement detection, transfer of the Binding Update [44] and the configuration of the new Care of Address (CoA) ) is performed only after the movement of the mobile node and being in the visited network, as it is used by MIPv6 [41] and "Proxy Mobile IPv6" (PMIPv6) [45].

- Proactive protocol: This kind of protocol involves performing mobility support as soon as the mobile node moves and before being disconnected from its attachment point, as it is used by Inter-PAN(2) [31], LoWMob, DLoWMob [32], Inter-Mario [39], Cluster-Based Scheme [40], FMIPv6 [42] and Inter-Mobility [46].

The proactive protocol is the most suitable for WSN with 6LoWPAN, since, it helps to reduce the handover delay by reducing the configuration time. It also helps to avoid the disconnection of nodes, which reduces the data loss rate. However, it requires an important processing and memory to find and predict the new attachment point of the mobile node. These disadvantages represent an important challenge to deal with.

To ensure a proactive process, the protocol must provide a rapid detection of the movement considering that the mobile node can move in a state of hibernation, and then predict the new attachment point of the mobile node [47][48].

\subsubsection{Movement detection}

Movement detection is a significant criterion to deal with the change in the attachment point of the mobile node. In WSN with 6LoWPAN, it is necessary to perform it in terms of providing minimum signaling cost, power consumption and handover delay. Hence, two main questions can be asked: Who is to perform the movement detection? and how to perform it? On the one hand, to ensure good performances in the signaling cost and the power consumption for the mobile node, it is not recommended that this entity execute the movement detection because of its limited resources. Hence, the movement can be detected by the edge router or other nodes in the network. On the other hand, to reduce the handover delay, this criterion of detection should be fast. In other words, the movement should be detected on time.

Following the state-of-the art, existing protocols use many ways to ensure the movement detection:

- A periodic sent of a Router Advertisement (RA) messages containing the prefix information [11]. The movement is detected in case this information changes.
- A periodic sent of a beacon having the PAN-ID information [49].

- A periodic sent of a Node Registration messages (NR) used by the Neighbor Discovery (ND) protocol [11][50][51] to check in the whiteboard table the existence of node address. The movement is detected when the source address of NR does not exist in this table.

- An estimation of the link quality based on Link Quality Index (LQI).

- The degradation of the Received Signal Strength Indicator (RSSI) value [52].

In WSN based on 6LoWPAN, the periodic sent of messages affects the signaling cost. In addition, RSSI cannot be well applied in indoor environment, because of the reflection problem of the used signals [53]. Thus, another method is needed for the movement detection.

\subsubsection{Mobility prediction}

Mobility prediction consists of predicting the new attachment point of the mobile node after its disconnection. The idea behind this concept is to reduce the time of the handover process, and then to improve the performance of the protocol. In order to introduce this prediction, the position of the mobile node, its direction and the positions of its neighbors are selected as parameters, and their assessment are based on some techniques such as the Received Signal Strength Indicator (RSSI) and the Angle of Arrival (AOA) [31][32][40][46].

\subsection{QoS consideration}

Ensure Quality-of-Service is important for most of applications, such as: providing high transfer data rate, little power consumption, more security services and low end-to-end delay.

\subsubsection{Data buffered}

During the handover process, the data transferred to the mobile node must be buffered in the home agent or the foreign agent, and be sent to the mobile node after confirming its new attachment point. This process allows to avoid data loss during the vulnerable handover period which is required to configure the new attachment [33].

\subsubsection{Duty cycle consideration}

Given the limited energy of sensors, nodes should alternate between active and inactive, called "Duty cycle" execution. This process is performed mainly when the node is in state of hibernation to preserve its power and extend its lifetime [15], as it is used by the Inter-PAN(2) [31].

\subsubsection{Security consideration}

Many eavesdroppers and attacker can find node location, send false node information and compromise its privacy and data confidentiality. For these reasons, it is necessary to provide security by ensuring protection, integrity and confidentiality of the resources [34]. Hence, mobility support protocol 
should use authentication and confidentiality, as it is used by HWSN6 [27][28][29], "Sensor Proxy Mobile IPv6" (SPMIPv6) [54][55]. However, in WSN based on 6LoWPAN, it's important to optimize the cost of security by taking into account the resources constraints [56][57][58].

\subsubsection{Routing optimization after a handover process}

After joining a visited network, when a correspondent node from the IP network wants to communicate with the Mobile Node (MN), data is sent to the Home Agent (HA). This one performs binding update between the two addresses of the mobile node (HoA and CoA). Then, it sends data to the Foreign Agent (FA), which transfers it to the MN. This is the case of the triangle routing [12], as it is used by HWSN6. However, this process increases the end-to-end delay of the communication between the source and the destination. Therefore, it is suitable to focus on the optimization of the route. Thus, data must be intercepted by the FA without passing through the home agent, as it is performed by MIPv6 [41].

\subsection{Kind of address}

Macro mobility in WSN with 6LoWPAN causes a change in the IPv6 address. However, providing a new IPv6 address follows some steps: Configuration, Duplicate Address Detection (DAD) process and registration. These steps affect the handover delay. For these reasons, dealing with mobility should take into consideration the used kind of address.

In order to reduce the handover delay, many proposed protocols discuss the used node address. For instance, PMIPv6 uses a fixed IPv6 address in its domain, since it uses a multi-homing process. So, it reduces the handover delay by avoiding the time needed for a new address reconfiguration and for the DAD process. On the other hand, the protocols [39][42] are based on the address pre-configuration process where the mobile node configures its Care-of-Address before reaching the visited network.

In WSN with 6LoWPAN standard, the node uses an IPv6 address in the outside of the network, which combines the prefix (64 bits) received from the edge router and the Interface Identifier IID (64 bit) configured by the node. In 6LoWPAN, the node uses a 16 bit short address generated by the edge router when the node joins the 6LoWPAN network in order to use less bits reserved for address.

\subsection{Topology architecture}

The functioning of the mobility support protocol depends on the topology architecture of the network, which is made according to the need of some applications.

WSN based on 6LoWPAN can be created following different topology architectures such as star topology, hierarchical topology based on tree configuration, mesh topology, grid topology, linear topology and many others. Nevertheless, considering the limited resources of this network type and to ensure a suitable mobility protocol support, some requirements have to be found in the chosen topology, as follows:

\subsubsection{Multi-hop consideration between the mobile node and the edge router}

In order to reduce power consumption, mobility support protocol should take into account the multi-hop communication from mobile node to the edge router, because the mobile node requires an important power consumption when it is too far from its communicating node.

\subsubsection{Local entity to deal with micro mobility}

This requirement was used in order to reduce the handover delay. According to research studies, some protocols such as "Distributed LoWMob" (DLoWMob) [32] and "Hierarchical Mobile IPv6" (HMIPv6) [59] use a special entity within the 6LoWPAN networks, which acts as a local gateway and manages mobility for a set of nodes, so as to reduce traffic control messages towards the global gateway (which conserve power for nodes in its vicinity) and reduce the handover delay for the micro mobility. Cluster-Based Scheme also performs this concept without using a special entity, but the ancestor parent node can be used as the responsible to deal with mobility in its subtree (without involving the gateway).

\subsubsection{Node deployment strategy}

To satisfy the functioning and role of applications, some nodes are deployed within the 6LoWPAN Network to monitor and track the mobile node. So, they must be deployed in such a way to provide coverage and connectivity in the entire area of interest to avoid data loss. Besides, nodes should be deployed considering a minimum number of active nodes, to reduce power consumption of nodes, like the process used in "Mobility-assisted minimum connected sensor cover" (MCSC) [60].

\section{Comparative study of existing mobility support proto- cols}

The work on mobility management was started in the 1990's. First propositions were based on routing protocol such as Cellular IP [61] and HAWAII [62]. These are a host based protocols, which require an active participation from the mobile node. Thus, the mobile node must periodically send control messages to achieve dealing with mobility. Therefore, this type requires a great signaling cost and power consumption for the mobile node, which it is not suitable with the constraint of WSN based on 6LoWPAN.

A suitable protocol dealing with mobility in WSN based on 6LoWPAN technology is delicate, because of its great number of nodes and its constrained resources in terms of power, bandwidth, memory, data rate and range. Hence, protocol must provide a satisfied quality of services considering the requirements of 6LoWPAN [15][63], namely a less power consumption (longer lifetime), less signaling cost, less handover delay, less end-to-end delay, avoid or reduce data loss, security and scalability. There are many mobility support protocols proposed to enhance some performances, however, each of them still has some drawbacks. 


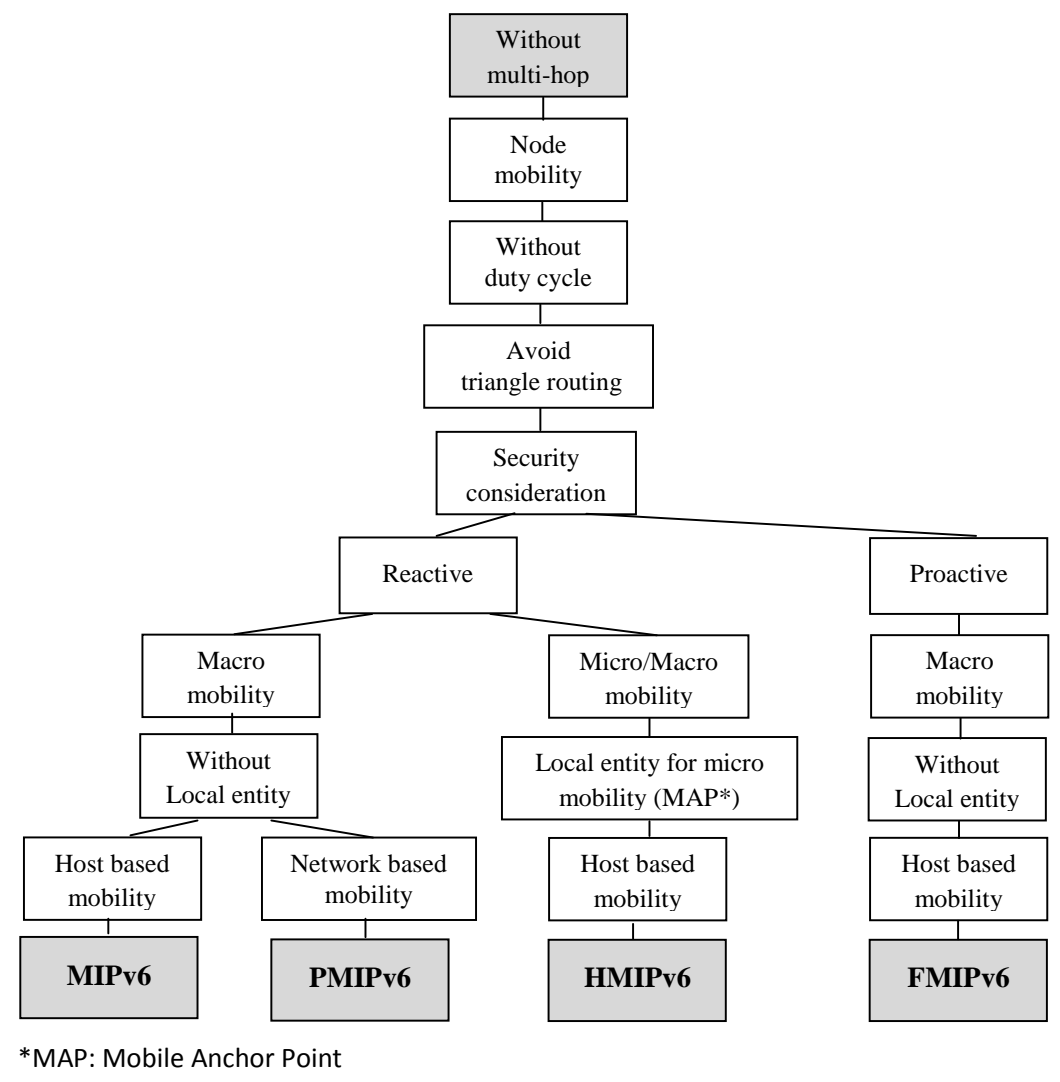

Figure 5: Classification of mobility support protocols for mobile IPv6 Networks

\subsection{Mobility support protocols for mobile IPv6 Networks}

In the early 2000's, some protocols were proposed for node mobility and macro mobility type, which attempted to improve some performances, such as "Mobile IPv6" (MIPv6) [11][41], "Fast Handover for Mobile IPv6" (FMIPv6) [42], "Proxy Mobile IPv6" (PMIPv6) [11][45] and "Hierarchical Mobile IPv6" (HMIPv6) [59]. The used criteria for these protocols is represented in Fig. 5, and its impact on the network performances is discussed in the following sections. Brief summarization of these protocols are shown in table 1 .

\subsubsection{Signaling cost and its impact on power consumption}

PMIPv6 [45] is a network based protocol whose the entity called Mobile Anchor Gateway (MAG) is the responsible for sending and exchanging messages related to the mobility support, instead of performing it by the mobile node as in MIPv6, FMIPv6 and HMIPv6. Therefore, PMIPv6 helps the mobile node to reduce its signaling cost, which reduces its power consumption [11].

Nevertheless, some problems appear when applying these protocols in the WSN based on 6LoWPAN, due to its strict constraints. First, these protocols use the prefix change to detect the movement by the mobile node. Thus, there is a periodic broadcast diffusion of Router Advertisement (RA) messages within the network, which increases the signaling cost and the power consumption. Second, they don't consider the multi-hop communication between the mobile node and its edge router, which requires great power consumption for the mobile node to communicate with its edge router when it is too far. Third, they use tunneling to buffer data and send it through the new attachment point. However, tunneling requires using a lot of control information by the mobile node, which increases signaling cost and power consumption. Fourth, they do not perform a duty cycle to save power when nodes are in state of hibernation.

\subsubsection{Handover delay}

PMIPv6 is based on the multi-homing concept that the Local Mobility Anchor (LMA) entity acts as a home agent for all the PAN Networks, which allows the mobile node to use a fixed IPv6 address in its domain, since the prefix remains the same. Hence, when the mobile node moves away from its home network, it does not need to configure a new care of address. Therefore, the handover delay is reduced because, it does not need any time to configure an address and perform the Duplicate Address Detection (DAD) [11].

Moreover, FMIPv6 and HMIPv6 concepts help to improve the handover delay. FMIPv6 uses a proactive process, that can anticipate the new care of address configuration of the mobile node before being disconnected from its home network. HMIPv6 also reduces the handover delay for the micromobility using a local entity within the network, to manage mobility for a set of nodes without involving the gateway. This entity, called Mobile Anchor Point (MAP), which acts as a local home agent to reduce the delay that occur during the message 
Table 1: Comparative study between different mobility support protocols for mobile IPv6 Networks

\begin{tabular}{|l|l|l|l|l|}
\hline & Address & $\begin{array}{l}\text { Movement } \\
\text { detection }\end{array}$ & $\begin{array}{l}\text { Data } \\
\text { buffered }\end{array}$ & $\begin{array}{l}\text { Topology } \\
\text { architecture }\end{array}$ \\
\hline MIPv6 [41][11] & IPv6 & RS/RA & HA & Star \\
\hline PMIPv6 [45][11] & Fixed IPv6 & RS/RA & LMA & Star \\
\hline HMIPv6 [59] & RCoA/LCoA & RS/RA & HA or MAP & Star \\
\hline FMIPv6 [42] & IPv6 & RS/RA & HA & Star \\
\hline
\end{tabular}

exchange.

\subsubsection{End-to-end delay}

The end-to-end delay is the necessary time to transmit packet across the Network from the source node to the destination node. After a handover process, packets may need more time for the end-to-end delay (as explained above in section 3.5.4). Here, MIPv6, HMIPv6 and FMIPv6 use the triangle routing only for the first packet between communicating nodes. Then, they can avoid it for the rest of packets, to ensure the same end-to-end delay before and after the handover process. Also, PMIPv6 can keep the same end-to-end delay, since it is based on a multi-homing concept, which avoids using the triangle routing after each movement.

\subsubsection{Security}

Security is considered by these Protocols. On the one side, MIPv6, HMIPv6 and FMIPv6 are based on the IPSec protocol to secure messages related to mobility (the binding update and the binding advertisement). On the other side, PMIPv6 uses a security architecture called "AAA" [54][55], which is responsible for Authentication, Authorization and Accounting of the mobile node.

According to the following, PMIPv6 appears the most appropriate to be applied in 6LoWPAN networks. However, it cannot be directly applied and it requires an important adaptation.

\subsection{Mobility support protocols for sensor Networks}

In the recent years, many efforts have been made for sensor Networks to support both mobility and routing, since most of the applications, which require the mobility of their nodes, affects routing path and cause data losses.

"Zone Routing Mobile Sensor Networks" (ZoroMSN) [64] is a distance based and hybrid (proactive and reactive) routing protocol supporting nodes mobility within the network (micro mobility). It is performed in an area divided into some equal zones containing a zone head, which acts as the router to forward data generated from its members towards the sink node through other zone heads. All the zone heads are organized in a tree topology. In addition, a new proposed protocol called "Mobility-assisted minimum connected sensor cover" (MCSC) [60] consists of collecting data and routing it to the sink node, using minimum active nodes which cover the entire area of interest. This area is divided into some grids representing a cluster including nodes organized in a tree topology and containing a cluster head, which is the responsible for forwarding data collected from the cluster members toward the sink node through other cluster heads. The MCSC supports micro mobility of a node to replace a failure node and maintain the area coverage. The used criteria of these two protocols is represented in fig. 6 , and their impact on network performances is discussed in the following sections. Brief summarization of these protocols are shown in table 2 .

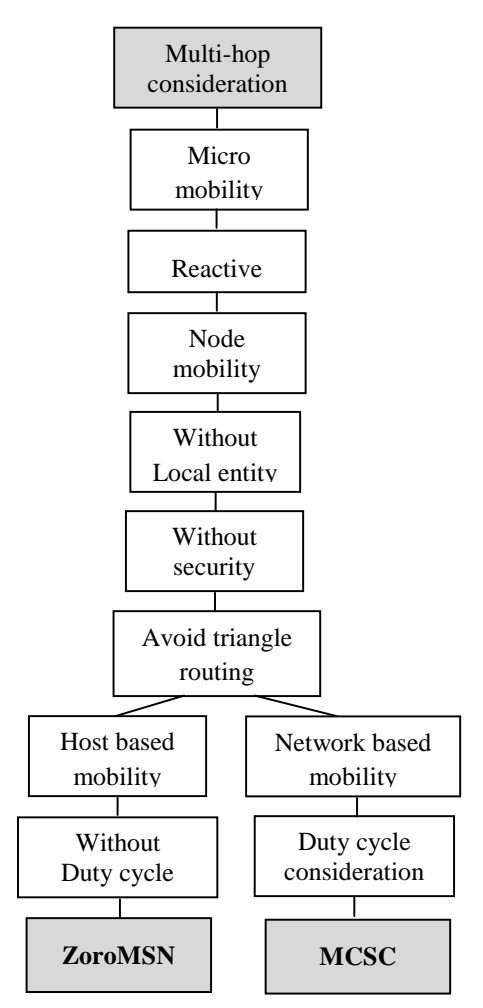

Figure 6: Classification of mobility support protocols for sensor Networks

\subsubsection{Signalling cost and its impact on power consumption}

ZoroMSN and MCSC follow a hierarchical routing within the network, which helps them to reduce the signaling cost and power consumption. In fact, the hierarchical routing avoids exchanging route request (RREQ) and route reply (RREP) messages between neighboring zone heads to discover a route path, and avoid the routing loop. In addition, these two protocols can conserve power of nodes through the multi-hop consideration to forward data. This can be achieved relying on the distribution of consumption among different zone heads.

Moreover, the power consumption for ZoroMSN was reduced and this was proved following the energy model, which 
Table 2: Comparative study between different mobility support protocols for sensor Networks

\begin{tabular}{|l|l|l|l|l|l|}
\hline & Address & Data buffered & $\begin{array}{l}\text { Topology } \\
\text { architecture }\end{array}$ & $\begin{array}{l}\text { Mobility } \\
\text { model }\end{array}$ & $\begin{array}{l}\text { Deployment of } \\
\text { static nodes }\end{array}$ \\
\hline ZoroMSN [64] & IPv6 & Zone Head & Cluster tree & Random walk [72] & Random in \\
\hline MCSC [60] & IPv6 & Not considered & Hybrid: tree-mesh & To failure node place & square zones \\
\hline
\end{tabular}

depends on the transmitted bits and the hop number [64]. In fact, reducing these two parameters through choosing the shortest path and the minimum signaling messages, the power consumption is decreased. Furthermore, the MCSC considers some parameters to choose the appropriate path with the minimum power consumption. The path is chosen following the highest benefit parameter, which is based on the remaining energy of the source node, the number of hops and the distance between the source node and its parents. When compared to other routing protocols, simulation results show that the ZoroMSN outperforms the others in term of energy consumption and nodes lifetime [64]. Also, the same results are found for the nodes lifetime even when the number of nodes in the networks is increased, which proves that this protocol ensures scalability. In addition, the same results of power consumption are found for the MCSC compared to ZoroMSN [64].

Nevertheless, ZoroMSN wastes power due to the periodic process for the reconfiguration of neighbor discovery, to create a list of zone heads used to the next hop in the data routing (time-based). This process needs a high signaling cost because of some messages exchange between neighbors. Furthermore, the MCSC wastes power during some processing steps such as computing a combination measurement to select active nodes, computing a benefit parameter to choose the appropriate path, and the periodic remaining energy computation performed by each node to check its level and detect its failure [60].

\subsubsection{Handover delay}

Neither the ZoroMSN nor MCSC evaluates the handover delay. However, the inaccessibility time during the handover process is not reduced, since there is no proactive concept to predict the new attachment of the mobile node with the zone head of the visited zone. Hence, the mobile node needs some delay to perform its configuration and join the zone as a member when it reaches it.

\subsubsection{End-to-end delay}

The end to end delay for the communication between each node and the sink node is well maintained by both the ZoroMSN and the MCSC protocols in case of a static network, since it performs a route optimization through choosing the lowest path and ensuring the free loop. Nevertheless, by introducing the mobility of some nodes, the link may break up and the data transmission will be affected. For instance, in the ZoroMSN, all zone heads in the Zone head list, which are used for the next hop, can move to other positions to be a member in another zone. In this case, the transmitter node will be unable to find the next hop to send its data, and thus it will buffer data and wait for the next neighbor discovery process to find another head which represent the next hop for the sink. This process increases the end to end delay. Even though, the MCSC reacts to solve this problem using a redundant node to recover a failure node, this process requires an extra time which affects the transmission delay.

\subsubsection{Data loss rate}

Mobility of nodes affects the route path and causes data loss. The ZoroMSN concept helps to reduce the data loss rate, through the route maintenance method and data buffering. In fact, when the zone head does not find any zone head on its list of neighbors for the next hop, it buffers data and waits for the next neighbor discovery process. In case of the mobility of a zone head which has data to send, this one changes its state becoming a member in the visited zone, then forwards the data buffered to its new zone head. Simulation results prove the decrease of data loss rate compared to other routing protocols supporting mobility. In addition, we noted that using large zones or high speed increases the data loss, which proves that this protocol is more adaptable to small zones with low mobility speed.

Furthermore, the failure of a node in the network causes an uncovered hole, which causes the partitioning of the network, disconnects the data transmission path and disturbs the functioning of the application used in the network. The MCSC deals with this problem to avoid the loss of the collected data. Hence, its concept consists in using redundant inactive nodes activated in case of active nodes failure. In fact, each node periodically checks its remaining energy level to detect its failure. This concept helps to avoid the uncovered holes, and then to ensure collecting and sending all the data.

\subsection{Mobility support protocols for 6LoWPAN networks with- out multi-hop consideration}

The "Hospital Wireless Sensor Networks" (HWSN6) [27][28][29] and the "Sensor Proxy Mobile IPv6" (SPMIPv6) [54][55] are two proposed protocols that deal with network mobility and micro/macro mobility for a healthcare application based on the 6LoWPAN networks. The main goal consists in tracking the patient, who can moves freely with some sensors node put in his clothes. Inter-Mario [39] and "Soft Handover for Mobile Wireless Sensor Networks" (SH-WSN6) [65] are protocols dealing with node mobility and macro mobility for the 6LoWPAN networks with some improvements. The used criteria of these two protocols are represented in fig. 7, and their impact on the network performances is discussed in the following sections. Brief descriptions of these protocols are shown in table 3 . 


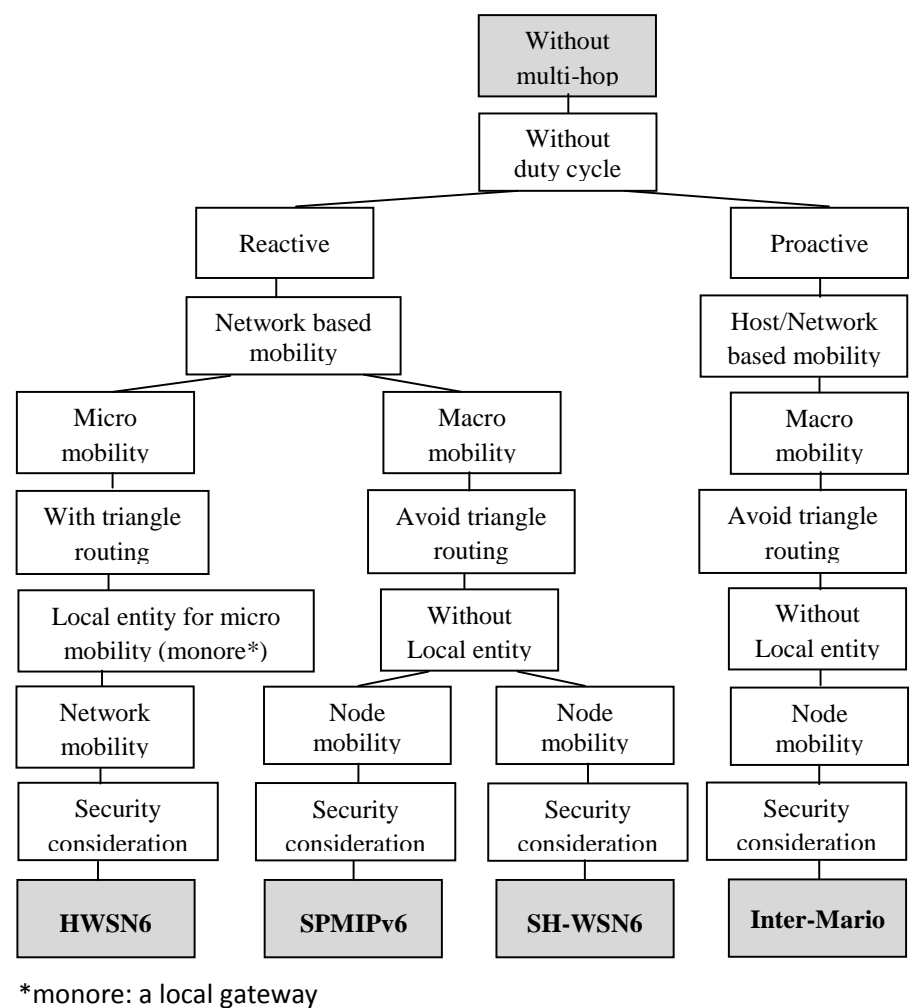

Figure 7: Classification of mobility support protocols for 6LoWPAN Networks without multi-hop consideration

Table 3: Comparative study between different mobility support protocolsfor 6LoWPAN Networks without multi-hop consideration

\begin{tabular}{|c|c|c|c|c|c|}
\hline & Address & $\begin{array}{c}\text { Movement } \\
\text { detection }\end{array}$ & $\begin{array}{c}\text { Data } \\
\text { buffered }\end{array}$ & $\begin{array}{c}\text { Topology } \\
\text { architecture }\end{array}$ & $\begin{array}{c}\text { Mobility } \\
\text { model }\end{array}$ \\
\hline HWSN6(1) [27] & Fixed IPv6 & PAN-ID & Not considered & Star & Unspecified \\
\hline HWSN6(2) [28][29] & Fixed IPv6 & NR/NC \& NS/NA & Not considered & Star & Unspecified \\
\hline SPMIPv6 [54][55] & Fixed IPv6 & RS/RA & SLMA & Star & $\begin{array}{l}\text { Probabilistic Random walk } \\
\text { based on Markovchain [73] }\end{array}$ \\
\hline Inter-Mario [39] & IPv6 & RSSI/link quality & FA & Star & Unspecified \\
\hline SH-WSN6 [65] & IPv6 & RS/RA & LMA & Star & Unspecified \\
\hline
\end{tabular}

\subsubsection{Signaling cost and its impact on power consumption}

SPMIPv6 can be applied in a hospital or at the patient's home. It is based on PMIPv6 by combining entity of Authentication with the LMA (the home agent) and messages of authentication with the Binding Update, which reduces the number of messages in the network, thus reducing the signaling cost.

In addition, HWSN6 and SPMIPv6 are a network based protocol for which the foreign agent for HWSN6 and the Mobile Access Gateway (MAG) for the SPMIPv6 are responsible to send the mobility signaling. This reduces the involvement of the mobile node, and thus its signaling cost and power consumption.

The evaluation and simulation results of SPMIPv6 prove that the signaling cost (number of bits of messages RS, RA and BU) increases as the number of hops and the number of nodes increase, since it will uses more signaling messages. The power consumption increases when the node density and data payload increase. In contrast, they are still lower when compared to
MIPv6 and PMIPv6 [54][55].

Nonetheless, these protocols still face some problems to conserve power. First, since they do not solve the multi-hop communication problem within the 6LoWPAN networks. Thus, the mobile node consumes more power to communicate with the gateway when it is too far. Second, SPMIPv6 and SHWSN6 still use the periodic broadcast of the RA messages to detect movement; and HWSN6 uses the change of the PANID received periodically in a beacon, or by the periodically sent NR (Node Registration) message by the mobile node to the gateway. These concepts require the involvement of the mobile node, which cause the overload of the bandwidth and increases the signaling cost and the power consumption in the network. Third, Inter-Mario increases the signaling cost through the double sending of the binding updates by the mobile node (host based protocol) and by the foreign agent in the pre-configuration process (network based protocol). Ultimately, they do not consider the duty cycle for nodes to con- 
serve power.

\subsubsection{Handover delay}

HWSN6 proposes a new architecture dedicated to be used in a hospital. Its concept consists in reducing the handover delay by using a local gateway in each room called "monore system", which is responsible to deal with the corresponding patient mobility.

Besides, HWSN6 and SPMIPv6 help to reduce the handover delay using a fixed IPv6 address, so they do not need a supplement time whether to configure a new care of address, or to perform a duplicate address detection during the movement process.

Moreover, Inter-Mario interests to reduce the handover delay based on MIPv6. To this end, it uses a proactive process performed with the help of a static node called "Partner Node" (PN) in the simple 6LoWPAN architecture. This process consists in carrying out monitoring and movement detection of nodes by computing the Received Signal Strength Indicator (RSSI), and in executing a pre-configuration of the future handover (before disconnection of the mobile node from its current attachment) through the exchange of information between the mobile node and the PANs in the vicinity with the help of the PNs. This process helps the mobile node to reduce the handover delay by scanning selectively the frequency of the PANs when it moves away from its home network, instead of scanning all the frequencies in the vicinity .

Then, following the state-of-the art, we noted that previously, the mobile node is attached only to one gateway and changes its attachment each time it receives a Router Advertisement message from a different gateway, which causes an unnecessary handover with the risk of losing connection. The solution concept of SH-WSN6 is based on idea to have more routes for the mobile node in order to ensure a continuous connectivity and to avoid handover process. It suggests to allow the mobile node to connect with more than one gateway and to have more IPv6 addresses, when there are more gateways in its range. This concept provides gain of a new route and improves connectivity. It also proposes to remove unreliable links using a comparing algorithm of the receiving Router Advertisement messages ratio, in order to improve Quality of Services (QoS) and ensure an acceptable end-to-end delay. According to the evaluation in [65], the handover delay of SH-WSN6 provides an acceptable results, but it is not the best solution to have the fastest handover.

Nonetheless, Inter-Mario cannot succeed in achieving its goal in every movement of the mobile node, mainly in a rapid movement. In this case, it will perform a MIPv6 operation. In addition, there is a tradeoff between the fast handover and the great signaling cost for the mobile node and the network. This tradeoff is proved by simulation of Inter-Mario [39]. When compared with PMIPv6, the handover delay (the sum of the forwarding delay) is noticeably lower with an increased number of hops, but its signaling cost (including routing cost, signaling cost of pre-configuration and binding updates) is higher. Furthermore, the reactive process of HWSN6 and SPMIPv6 (that they deal with mobility only after the mobile node reaches the visited network) increases the handover delay.

\subsubsection{End-to-end delay}

SPMIPv6, SH-WSN6 and Inter-Mario use the multi-homing concept to avoid the triangle routing after the handover process which allow to reduce the end-to-end delay. The simulation results of Inter-Mario in [39] shows that the route between nodes is optimized, and end-to-end delay is more stable. However, HWSN6 does not optimize the triangle routing process, because all data detected from the mobile patient must be transferred to its home gateway, which overload this gateway, and then it increases the end-to-end delay.

\subsubsection{Security}

Since HWSN6 and SPMIPv6 are designed to monitor vital constants and dependent on the patient's life applications [66], they introduce the security services such as the confidentiality, and the authentication of the mobile node. However, the cost of these security services is not taken into account to improve the network performance.

\subsection{Mobility support protocols for 6LoWPAN networks with multi-hop consideration}

Since, the major power consumption is caused by the deficit of the multi-hop communication within the 6LoWPAN networks, some protocols are proposed to resolve this problem and improve performances in the Networks. For instance, in [67], this issue is dealt by the combination between the proper concept of MIPv6 and the routing protocol OLSR (MIPv6+OLSR). Other proposed protocols ensure the multi-hop communication with the help of some static nodes deployed within the 6LoWPAN. These are responsible for tracking the mobile node and routing packets from/to it. Among these protocols, we cite Inter-PAN [30][31], "LoW Mobility" (LoWMob) [32], "Distributed LoWMob" (DLoWMob) [32], Inter-Mobility [46] and RPL-Weight [68] for node mobility and Cluster-Based Scheme [40] for network mobility. The used criteria of these protocols is represented in fig. 8, and its impact on the network performances is discussed in the following sections. Brief summarization of these protocols are shown in table 4 .

\subsubsection{Signaling cost and its impact on power consumption}

The power consumption is noticeably reduced for the mobile node for almost all the protocols cited above, since they consider the multi-hop communication between the mobile node and the gateway of its 6LoWPAN network. In addition, they are classified as network based protocols, for which another entity acts as the responsible to perform the messages exchange and to manage mobility, instead of the mobile node itself. This criterion decreases the power consumption and the signaling cost for the mobile node.

The simulation results of LoWMob and DLoWMob show that the signaling cost is not impacted by the speed of the mobile node, because it associates with the same number of static nodes and it requires the same number of mobility messages. In 


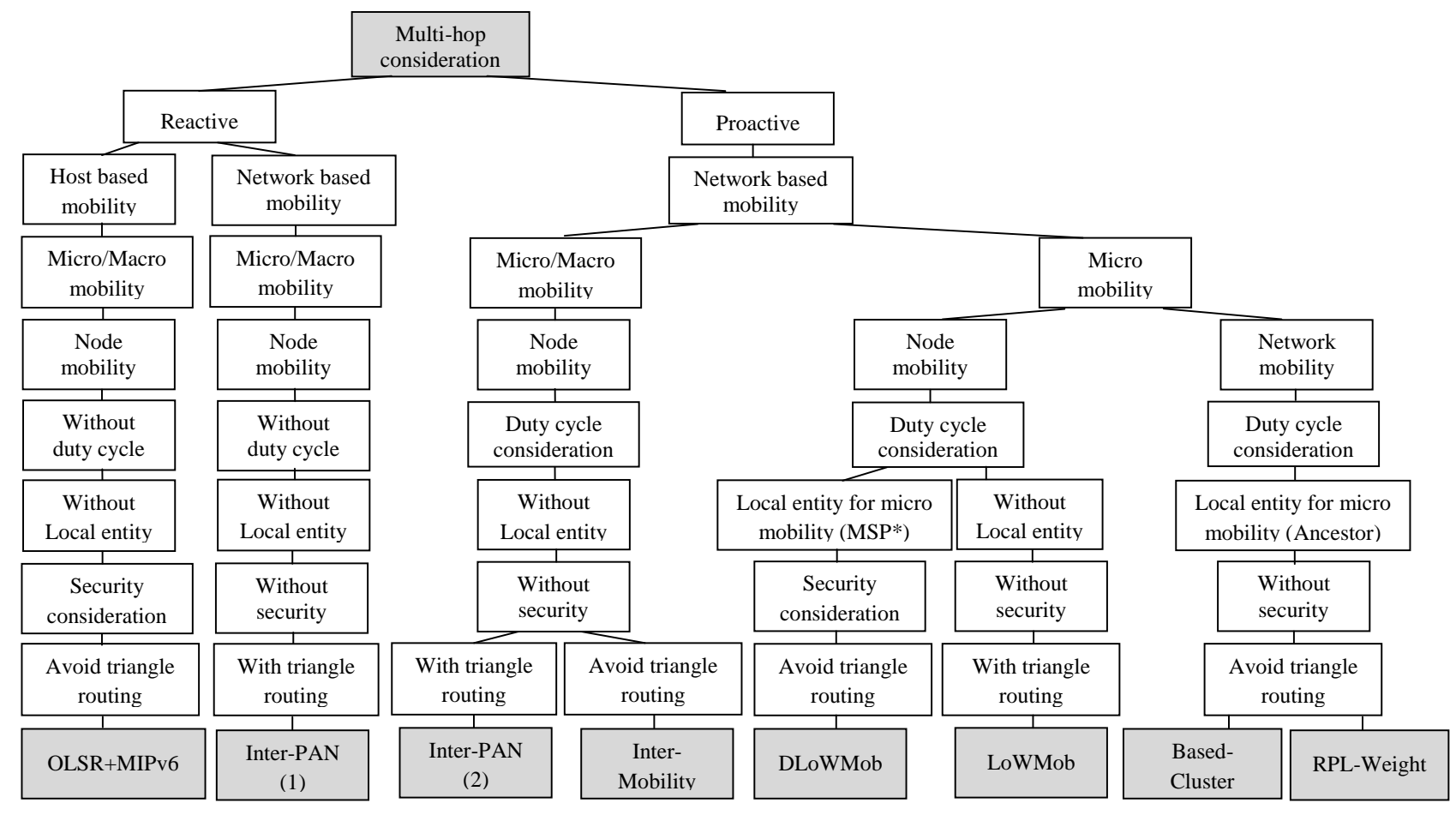

*MSP: Mobility Support Point

Figure 8: Classification of mobility support protocols for 6LoWPAN Networks with multi-hop consideration

addition, this performance parameter is better for DLoWMob than LoWMob, because the number of hops from the mobile node to the gateway is less important. Moreover, compared to HMIPv6, the signaling cost is lower, because it uses the packet size is optimized [32].

The simulation results of Inter-PAN(1) [30] show that the signaling cost does not change when the speed of the mobile node increases, because the mobile node associates with the same number of parent nodes, which provides the same number of messages. Contrary to Inter-PAN(2) [31], the signaling cost is reduced by the increasing speed. Because when the node speed is slower, the mobile node performs more handover due to sleep-active state transition of the static node [31]. This parameter is relatively reduced compared to the HMIPv6, given that this one performs more handover, which significantly impact more messages related to mobility.

Besides, the Cluster-Based Scheme protocol helps in the routing of the mobility control message by an automatic routing through the tree topology. Hence, there is no need to transmit control information to establish a routing path, which decreases the signaling cost. Simulation results of Cluster-Based Scheme [40] illustrate that the mobility handoff cost remains unchanged with an increasing speed, since there is the same number of associate nodes.

Unlike the previous protocols, the Routing Protocol for Low power and lossy networks-Weight (RPL-Weight) is based on routing protocol RPL which is able to manage micro mobility [68]. RPL is a hierarchical routing based on Directed Acyclic Graph (DAG) to define the network topology, and it uses Des- tination Oriented DAG (DODAG) algorithm. RPL-Weight is designed to track a mobile node with taking into account the sink node mobility. The sink node mobility contributes to reduce power consumption and to increase the network lifetime. Indeed, nodes close the sink are more asked to forward packets of other nodes addressed to the sink node. Therefore, the power consumption at these nodes is more important compared to other far nodes from the sink. Consequently, these nodes become unavailable rapidly which negatively impact the network lifetime. In order to mitigate this impact, and to increase network lifetime the load balancing policy can be introduced. In addition, RPL-Weight is a distributed based protocol which is not the case of other protocols based on the same concept.

Simulation results of RPL-Weight show that the networks lifetime is increased compared to the static sink, and it improves the network lifetime when the network size increases. Furthermore, RPL-Weight helps to reduce the signaling cost.

Nonetheless, MIPv6+OLSR increases the signaling cost and the power consumption within the networks, since it is a host based protocol and has the same concept as MIPv6. In addition, it does not maintain the duty cycle to conserve power for nodes.

\subsubsection{Handover delay}

LoWMob, DLoWMob, Inter-PAN(2), Inter-Mobility and Cluster-Based Scheme help to reduce the handover delay using the proactive process. This process is achieved by employing a parent static node for the mobile node, which acts as an anchor point. This entity concept consists in:

- Monitoring and detecting the movement of the mobile 
Table 4: Comparative study between different mobility support protocolsfor 6LoWPAN Networks with multi-hop consideration

\begin{tabular}{|c|c|c|c|c|c|c|}
\hline & Address & $\begin{array}{c}\text { Movement } \\
\text { detection }\end{array}$ & $\begin{array}{c}\text { Data } \\
\text { buffered }\end{array}$ & $\begin{array}{c}\text { Topology } \\
\text { architecture }\end{array}$ & $\begin{array}{c}\text { Mobility } \\
\text { model }\end{array}$ & $\begin{array}{c}\text { Deployment of } \\
\text { static nodes }\end{array}$ \\
\hline $\begin{array}{c}\text { OLSR+MIPv6 } \\
{[67]}\end{array}$ & IPv6 & RS/RA & HA & $\begin{array}{c}\text { Hybrid: } \\
\text { Mesh-Star }\end{array}$ & Unspecified & Random \\
\hline $\begin{array}{c}\text { LoWMob } \\
{[32]}\end{array}$ & $\begin{array}{c}\text { Out: IPv6 } \\
\text { In: 16-bit short }\end{array}$ & RSSI & PSN & $\begin{array}{c}\text { Hybrid: } \\
\text { Mesh-Bus }\end{array}$ & $\begin{array}{c}\text { Random } \\
\text { waypoint [74] }\end{array}$ & Grid \\
\hline $\begin{array}{c}\text { D-LoWMob } \\
{[32]}\end{array}$ & $\begin{array}{c}\text { Out: IPv6 } \\
\text { In: 16-bit short }\end{array}$ & RSSI & PSN & $\begin{array}{c}\text { Hybrid: } \\
\text { Mesh-Star-Bus }\end{array}$ & /Fluid flow [75] & $\begin{array}{c}\text { Random } \\
\text { in square zones }\end{array}$ \\
\hline $\begin{array}{c}\text { Inter-PAN(1) } \\
{[30]}\end{array}$ & $\begin{array}{c}\text { Out: IPv6 } \\
\text { In: 16-bit short }\end{array}$ & RSSI & GW & $\begin{array}{c}\text { Hybrid: } \\
\text { Mesh-Star-Bus }\end{array}$ & $\begin{array}{l}\text { Fluid flow based } \\
\text { on Marcov chain }\end{array}$ & Grid \\
\hline $\begin{array}{c}\text { Inter-PAN(2) } \\
{[31]}\end{array}$ & $\begin{array}{c}\text { Out: IPv6 } \\
\text { In: 16-bit short }\end{array}$ & RSSI & NPSN & $\begin{array}{c}\text { Hybrid: } \\
\text { Mesh-Star-Bus }\end{array}$ & $\begin{array}{c}\text { Fluid flow } \\
{[75]}\end{array}$ & Grid \\
\hline $\begin{array}{c}\text { Inter-Mobility } \\
{[46]}\end{array}$ & $\begin{array}{c}\text { Out: IPv6 } \\
\text { In: 16-bit short }\end{array}$ & $\begin{array}{c}\text { RSSI / } \\
\text { PAN-ID }\end{array}$ & $\begin{array}{c}\text { Intra: NPA } \\
\text { Inter: FA }\end{array}$ & $\begin{array}{c}\text { Hybrid: } \\
\text { Mesh-Star }\end{array}$ & Unspecified & Random \\
\hline $\begin{array}{c}\text { Cluster-Based } \\
\text { scheme [40] }\end{array}$ & Hierarchical & RSSI & $\begin{array}{l}\text { P. associated } \\
\text { node }\end{array}$ & $\begin{array}{c}\text { Hybrid: } \\
\text { Cluster tree-Bus }\end{array}$ & $\begin{array}{c}\text { Random } \\
\text { walk [76] }\end{array}$ & Grid \\
\hline $\begin{array}{c}\text { RPL-Weight } \\
{[68]}\end{array}$ & $\begin{array}{c}\text { IEEE } \\
802.15 .4\end{array}$ & $\begin{array}{c}\text { Intended } \\
\text { movement }\end{array}$ & $\begin{array}{l}\text { Sink } \\
\text { node }\end{array}$ & $\begin{array}{c}\text { Hybrid: } \\
\text { DoDAG-Mesh }\end{array}$ & $\begin{array}{l}\text { To computed } \\
\text { position place }\end{array}$ & Grid \\
\hline
\end{tabular}

node by a periodic computation of the RSSI value.

- Predicting the next localization of the mobile node based on RSSI computation and AOA techniques.

Furthermore, DLoWMob and Cluster-Based Scheme can help to reduce the handover delay for the micro mobility by avoiding the required delay to send signaling messages toward the gateway. Thus, DLoWMob uses an entity within the 6LoWPAN Networks called Mobility Support Point (MSP), which acts as a local gateway to deal with mobility within a set of nodes. In opposition, the Cluster-Based Scheme delegates to the common ancestor node to receive messages to deal with the node mobility within its sub-tree (without the involvement of the gateway). Moreover, RPL-Weight does not waste a handover delay since mobility is performed only during the global repair of its topology.

According to the simulation of the Cluster-Based Scheme [40], the handover delay relative to the increase in the number of hops is noticeably reduced compared to LoWMob, because this scheme performs mobility signaling only to the common ancestor node and it uses an automatic routing, which decreases delay to establish routing path.

\subsubsection{End-to-end delay}

Most of these protocols consider the route optimization after the handover process, which reduces the end-to-end delay of the communication data between the mobile node and its correspondent node.

According to the simulation results of LoWMob and DLoWMob [32], the end-to-end delay increases as the speed of the mobile node increases, because at a high speed, there are many interruptions of the association between the mobile nodes and their parents. In addition, simulation results show that the endto-end delay for DLoWMob is reduced up to twice compared to LoWMob.

\subsubsection{Security}

Only DLoWMob and MIPv6+OLSR introduce security services like confidentiality to secure the networks against eavesdroppers and attackers. DLoWMob using data encryption and authentication of the mobile node at the visited network. Moreover, MIPv6+OLSR uses IPSec to secure messages related to mobility.

\subsubsection{Data loss rate}

For LoWMob, DLoWMob, Inter-PAN, Inter-Mobility and Cluster-Based Scheme, when a parent static node detects a movement of the mobile node away from its range, it helps to buffer packets. Then, after the new attachment confirmation, it sends data buffered toward the next parent node to avoid data loss.

Simulation results of LoWMob and DLoWMob illustrate that at a high speed, the number of the handoffs increases, which causes data loss [32], so the packet success ratio is reduced when the mobile node increases its speed. In addition, the packet success ratio is better for DLoWMob, since the number of hops is lower. Moreover, simulation of the packet loss for Inter-PAN compared to HMIPv6 is reduced, given that HMIPv6 performs more handover with much delay, which causes data loss [30] [31]. Then, compared to the LoWMob, the ClusterBased Scheme simulation results show a less data loss [40], because of reducing the handover delay saves the number of the lost packets.

Ultimately, the Cluster-Based Scheme appears to be more suitable to the requirement of 6LoWPAN networks. Nevertheless, this scheme uses a hierarchical address depending on its topology organized in a tree architecture, that does not follow the 6LoWPAN standard. Neither does it consider dealing with macro mobility. Thus, more works are needed for management mobility in this kind of network. 


\section{Mobility in WSNs based on 6lowPAN: Future Directions}

Dealing with mobility in WSN based on 6LoWPAN technology is challenging issue, because of the strict constraints and the needed requirements of this network. Some important challenges (noted in section 2) must be taken into consideration to provide appropriate solutions for network with 6LoWPAN. In order to meet these challenges, and according to our analysis in the study made for the existing mobility support protocols, it is important to consider some directions and recommendations.

First, to be able to avoid data loss and increase the packet delivery ratio (PDR), mobility management protocols must reduce the bandwidth occupation which might be overloaded by the signaling messages. Besides, it must avoid the disconnection span time by performing the prediction process to anticipate problems and reduce the handover delay. The needed delay for the handover can be decreased by providing a continuous connectivity. Hence, it is crucial to perform a fast mobility detection with more accuracy [47][69], in order to rapidly find a new attachment point. Moreover, it is important to perform a fast handover, for instance by using a pre-configuration process of the mobile node address in the visited network, as it is used by Inter-Mario [39].

Second, to keep the same end-to-end delay as before and after the handover process and to reduce the jitter impact on the applications. It is necessary to optimize the triangle routing process. In addition, in WSN based on 6LoWPAN technology, it is important to reduce the frame fragmentation, so as to reduce the needed time to perform buffering and verifying of headers [27]. Hence, mobility management should optimize the payload size, and use signaling messages encapsulated in an IEEE 802.15.4 frames.

Third, to conserve the power of nodes and extend the network lifetime, which is considered as a key constraint of the WSN based on 6loWPAN technology, it is necessary to take into account some directions. For instance, performing duty cycle and topology control with consideration of multi-hop communication between nodes, since communication needs more power when the communicating nodes are too far from each other. Since the mobile node consumes more power then other nodes, it is preferable to apply a network based protocol. So, sending control messages should be performed by an entity other than the mobile node [43]. In addition, It is important to reduce treatments, since it requires power consumption and because of the limited resources in memory and processing. For these reasons, in WSN with 6LoWPAN, the protocol must reduce fragmentation, reduce signaling messages [33][43] and use compression mechanisms.

Fourth, security in WSN based on 6LoWPAN is already a challenging issue. In order to design a mobility management protocol, it is suitable to select the adapted cryptography algorithms to ensure security services with low cost from the link layer (IEEE 802.15.4) to the application layer. This point is recommended because performing a supplementary security mechanism requires more processing, memory and bandwidth, which are limited in this network type [27]. For this reason, it is recommended that the mobility support protocol should optimize the security cost according to the available resources (power, transmission rate,etc) [56][57][58]. In contrast, for the outside of the LoWPAN, the protocol may perform a mechanism [28] such as "IPSec" [70].

Fifth, it worth noting that the mobility management protocol must reduce complexity in terms of time, memory, and messages. In addition, the scalability is an important parameter which must be ensured.

Finally, it is recommended to have a glance at the IEEE 802.15.4g standard [71], since it has been used over the last few years to ease a large scale process control application (such as the smart grid). This standard can use multiple data rate in variable frequency bands, following different modes. For instance, the MR-OFDM "Multi-rate Orthogonal Frequency Division Multiplexing" was used to provide more data rate with higher spectral efficiency, "Multi-rate and multi-regional Offset Quadrature Phase Shift Keying" (MR-OQPSK) was applied to create multi-mode simple in design at a low cost, and "Multirate and multi-regional Frequency Shift Keying" (MR-FSK) was used to provide better transmission power efficiency. It's important to take into account the contribution of this standard in the conception and the evaluation of the mobility management protocol.

\section{Conclusion and perspectives}

Mobility of nodes in WSN with 6LoWPAN technology involves many advantages and functionalities for the needed applications. However, it represents a major challenge to cope with, because of its impacts and changes in this kind of network. In this paper, the state-of-art of mobility support protocols was surveyed. We began our work by introducing the challenges required to design a mobility management. Then, the classification criteria of mobility support protocols were proposed. The choice of such criteria depends on the used application and the needed performances. Based on these criteria, a comparative study of the existing protocols was presented to discuss the effect and impact of each used criterion on the performances of the 6LoWPAN networks.

After our study and analysis of the existing protocols, the major conclusion to be drawn is that there is no efficient solution to meet all the requirements and constraints of WSN with 6LoWPAN Technology. Thus, some improvements are still required. Moreover, the micro mobility was observed that it affect the routing path within the LoWPAN. Hence, micro mobility can't be treated without considering the routing protocol. IETF ROLL working group proposed a routing protocol for LoW Power and Lossy Networks called "RPL". This protocol might be considered in our future work to support mobility over the routing protocol in WSN with 6LoWPAN technology.

[1] Jennifer Yick, Biswanath Mukherjee, Dipak Ghosal, "Wireless sensor network survey", The International Journal of Computer and Telecommunications Networking, Vol. 52, Issue 12, pp. 2292-2330, 2008.

[2] I.F. Akyildiz, W. Su*, Y. Sankaras ubramaniam, E. Cayirci, "Wireless sensor networks: a survey", ELSEVIER Journal of Computer Networks, Vol. 38, pp. 393-422, 2002. 
[3] David Culler, Deborah Estrin and Mani Srivastava, "Overview of Sensor Networks", In IEEE journal of Computer Society, vol. 37, no. 8, pp 41-49, august 2004.

[4] K. Römer, F. Mattern, "The design space of wireless sensor networks", In IEEE Wireless Communications, Volume 11, Issue 6, pp: 54-61, ISSN: 1536-1284, December 2004

[5] Matt Welsh, David Malan, Breanne Duncan, Thaddeus Fulford-Jones, Steve Moulton, "Wireless Sensor Networks for Emergency Medical Care", Harvard University and Boston University School of Medicine, A talk presented at GE Global Research, March 8, 2004

[6] Stanković, Stanislava, "Medical Applications Based on Wireless Sensor Networks", Transactions Journal on Internet Research, Volume 5, Number 2, pp 19-23 (ISSN 1820-4503), July 2009.

[7] Ibtissam Boulanouar, Abderrezak Rachedi, Stéphane Lohier, Gilles Roussel, ¿'Energy-Aware Object Tracking Algorithm using Heterogeneous Wireless Sensor Networks" $\varnothing$ in 4th. IFIP/IEEE Wireless Days 2011 (IEEE WD’2011), Niagara Falls, Ontario, Canada, October 10-12, 2011. (IEEE Press)

[8] S.Prasanna, Srinivasa Rao, "An Overview of Wireless Sensor Networks Applications and Security", International Journal of Soft Computing and Engineering (IJSCE), ISSN: 2231-2307, Volume-2, Issue-2, May 2012.

[9] John A. Stankovic, Anthony D. Wood, Tian He, "Realistic Applications for Wireless Sensor Networks", Springer book in Theoretical Computer Science An EATCS Series, pp 835-863, ISSN 1431-2654, 2011.

[10] Antoine Bagula, "Application of Wireless Sensor Networks", A talk presented at WSN applications, UCT, feb 2012

[11] Zach Shelby, Carsten Bormann, "6LoWPAN: The Wireless Embedded Internet", book, Internet Engineering Task Force (6LoWPAN-WG), 2009.

[12] Luís M. L. Oliveira, Amaro F. de Sousa and Joel J. P. C. Rodrigues. "Routing and mobility approaches in IPv6 over LoWPAN mesh networks", International Journal of Communication Systems, Volume 24, Issue 11, pp 1445?1466, November 2011.

[13] Amitabha Ghosha, Sajal K. Dasb, "Coverage and connectivity issues in wireless sensor networks: A survey", Journal of Pervasive and Mobile Computing, Volume 4, Issue 3, pp 303-334, 2008.

[14] Benyuan Liu, Peter Brass, Olivier Dousse, Philippe Nain, Don Towsley, "Mobility Improves Coverage of Sensor Networks",The ACM International Symposium on Mobile Ad Hoc Networking and Computing "MobiHoc'05", Urbana-Champaign, Illinois, USA, May 25-27, 2005.

[15] Ricardo Nuno Mendo da Silva. "Mobility support in Low Power Wireless Personal Area Networks (MLoWPAN)". University of Coimbra, PORTUGAL, 2010.

[16] G. Montenegro, N. Kushalnagar, J. Hui, D. Culler, 'Transmission of IPv6 Packets over IEEE 802.15.4 Networks", Internet Engineering Task Force, IETF RFC 4944, September 2007.

[17] Jonathan Hui, David Culler, Samita Chakrabarti,"6LoWPAN: Incorporating IEEE 802.15.4 into the IP architecture", Internet Protocol for Smart Objects (IPSO) Alliance, Jan 2009.

[18] Md. Sakhawat Hossen, A. F. M. Sultanul Kabir, Razib Hayat Khan and Abdullah Azfar, "Interconnection between 802.15.4 Devices and IPv6: Implications and Existing Approaches", IJCSI International Journal of Computer Science Issues, Vol. 7, Issue 1, No. 1, January 2010.

[19] IEEE Computer Society Sponsored by the LAN/MAN Standards Committee, "Part 15.4: Wireless Medium Access Control (MAC) and Physical Layer (PHY) Specifications for Low-Rate Wireless Personal Area Networks (LR-WPANs)", IEEE Std 802.15.4€ oct 2003.

[20] Luca De Nardis and Maria-Gabriella Di Benedetto, "Overview of the IEEE 802.15.4/4a standards for low data rate Wireless Personal Data Networks", 4th Workshop on Positioning, Navigation and communication 2007 (WPNC'2007), Hannover, Germany, 2007.

[21] J.W. Hui, D.E. Culler, 'Extending IP to low-power, wireless personal area networks", IEEE Journal of Internet Computing, volume:12, issue:4, pp 37-45, ISSN: 1089-7801, July-Aug 2008.

[22] IEEE Computer Society. Part 15.4: "wireless medium access control (MAC) and physical layer (PHY) specifications for low-rate wireless personal area networks (WPANs)", IEEE Standard 802.15.4. August,2007.

[23] Kevin Dominik Korte, Iyad Tumar, Jrgen Schnwlder, "Evaluation of IPv6 over Low-Power Wireless Personal Area Networks Implementations", 4th IEEE International Workshop on Practical Issues In Building Sensor Network Applications (SenseApp 2009), Zurich, Switzerland; 20-23 October 2009.
[24] Nazim Abdeddaim, "Analyse des performances d'un rseau de capteurs exploitant le standard IEEE 802.15.4", thesis report, university of Grenoble, aug 2006.

[25] ZigBee Alliance, "ZigBee Specification", October 2007.

[26] Paolo Baronti, Prashant Pillai, Vince W.C. Chook, Stefano Chessa, Alberto Gotta , Y. Fun Hu, "Wireless sensor networks: A survey on the state of the art and the 802.15.4 and ZigBee standards", ELSEVIER Journal of Computer Communications, volume 30, issue 7, pp 1655-1695, may 2007.

[27] Antonio J. Jara, Miguel A. Zamora, Antonio F.G. Skarmeta, "HWSN6: hospital wireless sensor networks based on 6LoWPAN technology: mobility and fault tolerance management", IEEE International Conference on Computational Science and Engineering (CSE'09), Volume:2, pp 879884, Vancouver, BC, 29-31 Aug 2009.

[28] Antonio J. Jara, Miguel A. Zamora and Antonio F. G. Skarmeta, ”An Initial Approach to Support Mobility in Hospital Wireless Sensor Networks based on 6LoWPAN (HWSN6)", Journal of Wireless Mobile Networks, Ubiquitous Computing, and Dependable Applications, volume: 1, number: $2 / 3$, pp. $107-122,2010$

[29] Antonio J. Jara, Miguel A. Zamora, Antonio F.G. Skarmeta, "Intramobility for Hospital Wireless Sensor Networks based on 6LoWPAN", 6th IEEE International Conference on Wireless and Mobile Communications (ICWMC), pp 389-394, Valencia, 20-25 Sept 2010.

[30] Gargi Bag, Hamid Mukhtar, S.M. Saif Shams, Ki Hyung Kim, Seungwha Yoo, "Inter-PAN mobility support for 6LoWPAN", in Third International Conference on Convergence and Hybrid Information Technology, Busan, Korea, November 2008

[31] Gargi Bag, S.M. Saif Shams, Ali Hammad Akhbar, Muhammad Taqi Raza H.M, Ki-Hyung Kim and Seung-wha Yoo, "Network Assisted Mobility Support for 6LoWPAN", 6th IEEE Consumer Communications and Networking Conference, Las Vegas, USA, January 2009.

[32] Gargi Bag, Muhammad Taqi Raza, Ki-Hyung Kim and Seung-Wha Yoo, "LoWMob: Intra-PAN Mobility Support Schemes for 6LoWPAN", Journal of Wireless Sensor Technologies and Applications (Sensors 2009), Volume 9, Issue 7, pp 5844-5877, July 2009.

[33] N. Kushalnagar, G. Montenegro, C. Schumacher, "IPv6 over Low-Power Wireless Personal Area Networks (6LoWPANs): Overview, Assumptions, Problem Statement, and Goals", Internet Engineering Task Force, IETF RFC 4919, August 2007.

[34] J.Kempf, B.DoCoMo "Problem Statement for Network-Based Localized Mobility Management (NETLMM)", Internet Engineering Task Force, IETF RFC 4830, April 2007.

[35] Benyuan Liu, Peter Brass, Olivier Dousse, Philippe Nain, Don Towsley, "Mobility Improves Coverage of Sensor Networks", The ACM International Symposium on Mobile Ad Hoc Networking and Computing "MobiHoc'05", Urbana-Champaign, Illinois, USA, May 25-27, 2005.

[36] SILVIA DE LUCIA, "Optimization of Handover Algorithms for Wireless Networks", Master's Degree Project, Stockholm, Sweden, 2010.

[37] You-Chiun Wang, Fang-Jing Wu, and Yu-Chee Tseng, "Mobility Management Algorithms and Applications for Mobile Sensor Networks", WILEY of WIRELESS COMMUNICATIONS AND MOBILE COMPUTING, in Special Issue of Recent Advancement in Wireless Ad Hoc and Sensor Networks, Volume 12, Issue 1, pages 7?21, January 2012.

[38] M.Pascoe, J.Gomez, V.Rangel, M.Lopez-Guerrero, F.Mendoza, "A Mobility-Based Upper Bound on Route Length in MANETs", Springer Journal of Telecommunication Systems, Volume 52, Issue 1, pp 105-119, Jan 2013.

[39] Minkeun Ha, Daeyoung Kim, Seong Hoon Kim, Sungmin Hong, ’InterMARIO: a fast and seamless mobility protocol to support Inter-PAN handover in 6LoWPAN", IEEE Global Telecommunications Conference (GLOBECOM 2010), pp 1-6, Miami, FL, Dec 2010.

[40] Wang Xiaonan, Zhong Shan, Zhou Rong, ”A mobility support scheme for 6LoWPAN", ELSEVIER journal of Computer Communications, Volume 35, Issue 3, pp 392?404, February 2012.

[41] D. Johnson, C. Perkins, J. Arkko, "Mobility Support in IPv6", Internet Engineering Task Force, IETF RFC 3775, June 2004.

[42] R. Koodli, "Mobile IPv6 Fast Handovers", Internet Engineering Task Force, IETF RFC 5568, June 2008.

[43] Myung-Ki Shin, Hyoung-Jun Kim, "L3 Mobility Support in Large-scale IP-based Sensor Networks (6LoWPAN)", 11th IEEE International Conference on Advanced Communication Technology (ICACT 2009), Vol- 
ume 2, pp 941-945, Phoenix Park, 15-18 Feb 2009

[44] R. Silva and J. Sa Silva "An Adaptation Model for Mobile IPv6 support in LoWPANs", Internet Engineering Task Force, Internet-Draft draft-silva6lowpan-mipv6-00, May 2009.

[45] S.Gundavelli, K.Leung, V.Devarapalli, K.Chowdhury and B.Patil, "Proxy Mobile IPv6", Internet Engineering Task Force, RFC 5213, August 2008.

[46] Zinon Zinonos and Vasos Vassiliou, "Inter-Mobility Support in Controlled 6LoWPAN Networks", IEEE Conference of Ubiquitous Computing and Networks (Globecom 2010), pp 1718-1723, Miami, FL, 6-10 Dec 2010.

[47] Gargi Bag, Muhammad Taqi Raza H. M, Hamid Mukhtar, Ali Hammad Akbar, S.M. Saif Shams, Ki-Hyung Kim, Seung-wha Yoo and Donghwa Kim, "Energy-Aware and Bandwidth-Efficient Mobility Architecture for 6LoWPAN", IEEE Military Communications Conference (MILCOM 2008), pp 1-7, San Diego, USA, November 2008.

[48] Qian Dong, Waltenegus Dargie, "A Survey on Mobility and MobilityAware MAC Protocols in Wireless Sensor Networks", IEEE Journal of Communications Surveys and Tutorials, Volume 15, NO 1, 2013.

[49] Laurent Toutain, Kevin Perros, Joongsoo Lee, "Supprimer le protocole Neighbor Discovery dans les réseaux de capteurs", Colloque Francophone sur l'Ingénierie des Protocoles (CFIP), les Arcs : France, 2008

[50] Z.Shelby, P.Thubert, J.Hui, S.Chakrabarti, C.Bormann and E.Nordmark, "6LoWPAN Neighbor Discovery", Internet Engineering Task Force, Internet-Draft draft-ietf-6lowpan-nd-06, September 2009.

[51] Wikipedia-6LoWPAN. [En ligne] http://fr.wikipedia.org/wiki/6LoWPAN

[52] Andrew Attwood, Madjid Merabti, Omar Abuelmaatti, "Network Mobility and Fragmentation in Wireless Mesh Internetworks: Issues and Challenges", The 11th annual postgraduate symposium on the convergence of telecommunications, networking and broadcasting (PGNet), Network Mobility and Fragmentation in Wireless Mesh Internetworks: Issues and Challenges, june 2010.

[53] Hirokazu Miura, Kazuhiko Hirano, Noriyuki Matsuda, Hirokazu Taki, Norihiro Abe, Satoshi Hori, "Indoor Localization for Mobile Node Based on RSSI", Springer book: Knowledge-Based Intelligent Information and Engineering Systems, Volume 4694, pp 1065-1072, 2007.

[54] Md. Motaharul Islam, Mohammad Mehedi Hassan, Eui-Nam Huh, "Sensor Proxy Mobile IPv6 (SPMIPv6) - A Framework of mobility supported IP-WSN", Proceedings of 13th International Conference on Computer and Information Technology (ICCIT 2010), in Dhaka, Bangladesh, December, 2010

[55] Md. Motaharul Islam, Eui-Nam Huh, "Sensor proxy mobile IPv6 (SPMIPv6)- A novel scheme for mobility supported IP-WSNs Sensors", Sensor 2011, Volume 11, Issue 2, pp 1865-1887, 2011. //I/I/I/I/I/I

[56] Abderrezak Rachedi, Amina Hasnaoui, "Security with Quality-ofServices Optimization in Wireless Sensor Networks", in the IEEE The 9th International Wireless Communications \& Mobile Computing Conference (IWCMC'2013), Cagliari, Italy, July 1st-5th 2013.

[57] Abderrezak Rachedi, Lamia Kaddar, Ahmed Mehaoua, 'EDES- Efficient Dynamic Selective Encryption Framework to Secure Multimedia Traffic in Wireless Sensor Networks", in the IEEE International Conference on Communications (IEEE ICC'2012), pp. 1026 - 1030 , Ottawa, Canada, June 10-15, 2012

[58] Abderrezak Rachedi and Hend Baklouti, "muDog: Smart Monitoring Mechanism for Wireless Sensor Networks based on IEEE 802.15.4 MAC", in the IEEE International Conference on Communications (ICC'2011), Kyoto, Japan, 5-9 June 2011.

[59] H. Soliman, C. Castelluccia, K. ElMalki, L. Bellier, "Hierarchical Mobile IPv6 (HMIPv6) Mobility Management", Internet Engineering Task Force, RFC 5380, October 2008

[60] Ahmed M.Khedr, Walid Osamy, "Mobility-assisted minimum connected cover in a wireless sensor network", ELSEVIER Journal of Parallel and Distributed Computing, Volume 72, Issue 7, pp 827-837, July 2012.

[61] D. Saha, A. Mukherjee, I.S. Misra, M. Chakraborty, "Mobility Support in IP: A Survey of Related Protocols", IEEE Journal of Network, Volume 18, Issue 6, pp 34-40, 2004.

[62] R. Ramjee, T. La Porta, S. Thuel, K. Varadhan, S. Wang, "HAWAII: A Domain Based Approach for Supporting Mobility in Wide Area Wireless Area Networks", In Seventh International Conference on Network Protocols, pp 283-292, Toronto, Canada, 1999.

[63] Ricardo Silva, Jorge S Silva and Fernando Boavida, "Towards Mobil- ity Support in Wireless Sensor Networks", CRC'10, Braga, Portugal, oct 2010.

[64] Nidal Nasser, Anwar Al-Yatama, Kassem Saleh, "Zone-based routing protocol with mobility consideration for wireless sensor networks", Springer Journal of Telecommunication Systems, Volume 52, Issue 4, pp 2541-2560, April 2013.

[65] Juha Petäjäjärvi and Heikki Karvonen,'Soft Handover Method for Mobile Wireless Sensor Networks Based on 6LoWPAN", IEEE International Conference on Distributed Computing in Sensor Systems and Workshops (DCOSS), pp 1-6, Barcelona, 27-29 June 2011.

[66] Jin Ho Kim, Rim Haw, and Choong Seon Hong, "Development of a Framework to Support Network-based Mobility of 6LoWPAN Sensor Device for Mobile Healthcare System", International Conference on Consumer Electronics (ICCE), pp 359-360, Las Vegas, NV, 9-13 Jan 2010.

[67] Volker Köster, Dennis Dorn, Andreas Lewandowski and Christian Wietfeld, "A novel approach for combining Micro and Macro Mobility in 6LoWPAN enabled Networks", IEEE Vehicular Technology Conference (VTC Fall), pp 1-5, San Francisco, CA, 5-8 Sept 2011.

[68] L.B. Saad, B. Tourancheau, "Sinks mobility strategy in IPv6-based WSNs for network lifetime improvement", in International Conference on New Technologies, Mobility and Security IFIP (NTMS 2011), Paris, France, feb 2011.

[69] Damien Roth, Julien Montavont et Thomas Nol, "gestion de la mobilité à travers différents réseaux de capteurs sans fil", 12mes Rencontres Francophones sur les Aspects Algorithmiques de Télécommunications (AlgoTel), Belle Dune-Cote d'Opale, 31 May?3 Jun 2010.

[70] S. Kent, K. Seo, ”Security Architecture for the Internet Protocol”, Internet Engineering Task Force, RFC 4301, dec 2005.

[71] Karan S. Panchal, "Implementing Physical Layer (PHY) of IEEE 802.15.4G Standard with Direct Sequence Spread Spectrum (DSSS) Using Offset Quadrature Phase Shift Keying (O-QPSK)", thesis report of Master of Science, San Diego State University, 2012

[72] Navid Hassanzadeh, "Scalable data collection for Mobile Wireless Sensor Networks", thesis report of Master of Science, Stockholm, Sweden, novembre 2011

[73] Tracy Camp, Jeff Boleng, Vanessa Davies, "A survey of mobility models for Ad Hoc Network Research", Wireless Communication and Mobile Computing (WCMC): Special issue on Mobile Ad Hoc Networking: Research, Trends and Applications, Volume 2, Issue 5, pp 483-502, 2002.

[74] Alexander Pelov, "Mobility models for Wireless Networks", thesis report of computer science, university of Strasbourg, 2009.

[75] Christian Schindelhaue, "Mobility in Wireless Networks", In 32nd Annual Springer Conference on Current Trends in Theory and Practice of Informatics, (SOFSEM 2006), LNCS 3831, pp 100-116, Merin, Czech Republic, January 21-27, 2006.

[76] Cholatip Yawut, "Adaptation la mobilit dans les rseaux ad hoc", thesis report of computer science, university of Toulouse, 2009. 